\title{
EXTREMAL PROPERTIES OF GREEN FUNCTIONS AND A. WEITSMAN'S CONJECTURE
}

\begin{abstract}
ALEXANDER FRYNTOV
ABstract. A new version of the symmetrization theorem is proved. Using a modification of the *-function of Baernstein we construct an operator which maps a family of $\delta$-subharmonic functions defined on an annulus into a family of subharmonic functions on an annular sector. Applying this operator to the Green function of special domains we prove A. Weitsman's conjecture linked with exact estimates of the Green functions of these domains.
\end{abstract}

\section{INTRODUCTION}

Let $u(z)$ be an upper semicontinuous function defined on some domain $W$ of the complex plane $\mathbb{C}$. The function $u(z)$ is called subharmonic in the domain $W$ if for every $z \in D$ there exists $\rho_{0}>0$ such that the inequality

$$
u(z) \leq \frac{1}{2 \pi} \int_{0}^{2 \pi} u\left(z+\rho e^{i \theta}\right) d \theta
$$

is valid for every positive $\rho<\rho_{0}$. A function which admits the representation as a difference of two subharmonic functions is called a $\delta$-subharmonic function in this domain. The estimates of subharmonic functions are extremely useful for investigation of the behavior of analytic functions due to the following property: $\log |f(x)|$ is subharmonic function for every analytic function $f$.

For every function $u(z)$ subharmonic in the domain $W$ there exists a nonnegative measure $\mu$ such that for every domain $K, \operatorname{clos}(K) \subset W$, the Riesz representation holds:

$$
u(z)=u_{0}(z)+\int_{K} \log |z-\zeta| d \mu(\zeta),
$$

where $u_{0}(z)$ is harmonic inside $K$. The measure $\mu$ is called a Riesz measure of the subharmonic function $u(z)$. Other useful properties of subharmonic functions can be found, for example, in the book of Hayman and Kennedy [4].

Let $\Omega$ be a domain in the complex plane $\mathbb{C}$ and suppose that the following conditions are fulfilled:

(1) $1 \in \Omega$;

(2) every closed arc of angular measure $2 l(0<l<2 \pi)$ of every circle with center zero intersects the set $\mathbb{C} \backslash \Omega$.

Received by the editors March 17, 1992.

1991 Mathematics Subject Classification. Primary 31A05; Secondary 30C75.

Key words and phrases. Subharmonic function, Green function, symmetrization. 
Let $G_{\Omega}(z, \xi), z \in \Omega, \xi \in \Omega$, be the Green function of the domain $\Omega$ (with respect to the solution of the Dirichlet problem for Laplace's equation in this domain). We assume that the domain of definition of a Green function is the whole two-dimensional complex plane $C \times \mathbb{C}$ and $G(z, \xi)=0$ for $(z, \xi) \notin \Omega \times \Omega$. Such a definition transforms the Green function $G_{\Omega}(z, \xi)$ into a subharmonic one with respect to $z \in \mathbb{C} \backslash\{\xi\}$ for every fixed $\xi \in \mathbb{C}$.

The question of Weitsman [5] is the following: Let $\Omega$ be a domain satisfying conditions (1), (2), and let $\Omega_{0}$ be the sector $\{z: \arg (z) \in(-l, l)\}$. Is it true that the inequalities

$$
\begin{gathered}
\max _{|z|=r} G_{\Omega}(z, 1) \leq \max _{|z|=r} G_{\Omega_{0}}(z, 1), \\
\int_{-\pi}^{\pi} G_{\Omega}\left(r e^{i \varphi}, 1\right) d \varphi \leq \int_{-\pi}^{\pi} G_{\Omega_{0}}\left(r e^{i \varphi}, 1\right) d \varphi,
\end{gathered}
$$

for the Green functions are valid?

An affirmative answer to this question will be given here. A theorem about the subharmonic operators of (*)-type of Baernstein will be proved as well. The exact estimates in Weitsman's conjecture will be obtained using this theorem. We hope also that this theorem will be interesting even without its application to the proof of Weitsman's conjecture. Before formulation of this theorem we introduce some notions and definitions.

Let $u(z)$ be a $\delta$-subharmonic function defined in the annulus $\left\{|z| \in\left(r_{1}, r_{2}\right)\right\}$. Let

$$
u(z)=u_{+}(z)-v(z)
$$

be one of its representations as a difference of two subharmonic functions. Let $\left\{z=r e^{i \theta}: r \in\left(r_{1}, r_{2}\right), \theta \in(0, l)\right\}$ be an annular sector.

We define the function $u^{*}$ in this annular sector by the formula

$$
u_{i}^{*}\left(r e^{i \theta}\right)=\sup \left\{\int_{E} u\left(r e^{i \varphi}\right) d \varphi: E \in \mathscr{E}(\theta, l)\right\}
$$

Here. $\mathscr{E}(\theta, l)$ is the family of all measurable sets of the real axis that satisfy the conditions

(a) $\operatorname{mes}(E)=|E|=2 \theta$;

(b) $\operatorname{diam}(E) \leq 2 l$.

(Condition (b) means that there exists an interval $I$ such that $|I|=2 l$ and $E \subseteq I$.)

Now we make a few remarks about some useful properties of the function $u_{i}^{*}(z)$.

(1) The function $u_{l}^{*}(z)$ is continuous in the domain $\left\{z=r e^{i \theta}: r \in\left(r_{1}, r_{2}\right)\right.$, $\theta \in[0, l]\}$.

(2) $u_{i}^{*}(r)=0$ for $r \in\left(r_{1}, r_{2}\right)$;

(3) For every $z=r e^{i \theta}$ belonging to the domain of definition of the function $u_{l}^{*}(z)$ there exists $E \subset \mathscr{E}(\theta, l)$ such that

$$
u_{l}^{*}\left(r e^{i \theta}\right)=\int_{E} u\left(r e^{i \varphi}\right) d \varphi
$$

(i.e., the supremum in definition (2) is attained on some set $E \in$ $\mathscr{E}(\theta, L))$. 
We do not prove these properties but note that their proofs are the same as in Baernstein [1].

Let $\nu$ be the Riesz measure of a subharmonic function $v(z)$. Let

$$
V_{\rho}\left(r e^{i \omega}\right)=\int_{\xi \in C} \log ^{+} \frac{\rho}{\mid \xi-r e^{i \omega \mid}} d \nu(\xi)
$$

be the value of its Green potential with respect to the domain $\{\xi \in \mathbb{C}: \mid \xi-$ $\left.r e^{i \omega} \mid<\rho\right\}$ at $z=r e^{i \omega}$. We will say that this function is a local potential of the subharmonic function $v(z)$. According to the Poisson-Jensen formula (see, for example, [4]) the local potential of the function $v(z)$ can be defined by

$$
V_{\rho}(z)=\frac{1}{2 \pi} \int_{-\pi}^{\pi} v\left(z+\rho e^{i \psi}\right) d \psi-v(z)
$$

without using the measure $\nu$. We say that the subharmonic function $v(z)$ has a bell-type potential if for every $r \in\left(r_{1}, r_{2}\right)$ there exists $\rho_{0}>0$ such that for all $\rho<\rho_{0}$ the function $V_{\rho}\left(r e^{i \omega}\right)$ is even for $\omega \in(-\pi, \pi)$ and does not increase for $\omega \in(0, \pi)$.

Useful examples of bell-type potentials are the following:

Example 1. Let $\nu$ be a uniform distribution (i.e., $\nu$ coincides with Lebesgue measure) on the circle $\{z:|z|=R\}$. It is not difficult to see that the function

$$
V_{\rho}\left(r e^{i \omega}\right)=\frac{1}{2 \pi} \int_{-\pi}^{\pi} \log ^{+} \frac{\rho}{\left|R e^{i \lambda}-r e^{i \omega \mid}\right|} d \lambda,
$$

does not depend on $\omega$ so the local potential of the measure $\nu$ is bell-type.

Example 2. Let $\nu$ be the Dirac measure concentrated in the point $\xi=1$. It is easy to see that in this case the potential

$$
V_{\rho}\left(r e^{i \omega}\right)=\log ^{+} \frac{\rho}{\mid 1-r e^{i \omega \mid}}
$$

is bell-type.

Now we formulate the main theorem about *-transforms that is a new version of the symmetrization theorem of Baernstein [1].

Theorem 1. Let $u(z)$ be a $\delta$-subharmonic function represented by (1) in the annulus $\left\{|z| \in\left(r_{1}, r_{2}\right)\right\}$. If the local potential of $v(z)$ is bell-type, then the function

$$
u_{i}^{*}\left(r e^{i \theta}\right)+\int_{-\theta}^{+\theta} v\left(r e^{i \varphi}\right) d \varphi
$$

is continuous and subharmonic in the annular sector $\left\{z=r e^{i \varphi}: r \in\left(r_{1}, r_{2}\right), \theta \in\right.$ $(0, l)\}$.

This theorem is a refined version of the theorem obtained by the author [3] and by Baernstein [2] in order to prove the Levin conjecture about extremal values of the special class of subharmonic functions on the plane. Now we remark about an interesting property of a function $u_{i}^{*}(z)$. This property is essential for our applications of Theorem 1.

Remark 1. Let $u\left(r e^{i \varphi}\right) \geq 0$. If $\operatorname{mes}\left(I \cap\left\{\varphi: u\left(r e^{i \varphi}\right)=0\right\}\right)>0$ for every $r \in\left(r_{1}, r_{2}\right)$ and for every interval $I$ with $\operatorname{diam}(I) \geq 2 l$, then the function 
$u_{i}^{*}\left(r e^{i \theta}\right)$ does not depend on $\theta$ in some complex neighborhood of the set $\{z=$ $\left.r e^{i \theta}: r \in\left(r_{1}, r_{2}\right), \theta=l\right\}$.

To prove Theorem 1 we need the following lemma.

Lemma 1. Let $0<\theta<l$ and $E \in \mathscr{E}(\theta, l)$. For every sufficiently small $\delta \leq$ $\delta_{0}(E)$ there exist measurable sets $A \in \mathscr{E}(\theta+\delta, l)$ and $B \in \mathscr{E}(\theta-\delta, l)$ such that the equality

$$
\chi_{E}(t+\delta)+\chi_{E}(t-\delta)=\chi_{A}(t)+\chi_{B}(t)
$$

holds almost everywhere on $\mathbf{R}$. Here we denote by $\chi_{D}$ the indicator of $D$, i.e., the function

$$
\chi_{D}(t)= \begin{cases}1, & t \in D, \\ 0, & t \notin D .\end{cases}
$$

This lemma was used in [1] and [2]. One can also find its proof in $\S 4$. Now we prove Theorem 1.

\section{ProOf Of TheOREM 1}

According to property (1) the function $u_{i}^{*}(z)$ is continuous. To prove Theorem 1 it is sufficient to verify the integral inequality

$$
u_{l}^{*}(z)+\tilde{v}(z) \leq \frac{1}{2 \pi} \int_{-\pi}^{\pi}\left\{u_{l}^{*}\left(z+\rho e^{i \psi}\right)+\tilde{v}\left(z+\rho e^{i \psi}\right)\right\} d \psi
$$

for every $z$ from the domain of definition of the function $u_{i}^{*}(z)$ and for all sufficiently small $\rho<\rho_{0}(z)$. Here the function $\tilde{v}(z)$ for $z=r e^{i \theta}$ is defined by the equation

$$
\tilde{v}\left(r e^{i \theta}\right)=\int_{-\theta}^{\theta} v\left(r e^{i \varphi}\right) d \varphi .
$$

Now we prove the latter inequality. It follows from subharmonicity of the function $u_{+}(z)$ that for every fixed $z$ and for all $\rho>0$ such that $|z| \pm \rho \in$ $\left(r_{1}, r_{2}\right)$ the inequality $\left(z=r e^{i \omega}\right)$

$$
\begin{aligned}
u_{+}(z) & =u(z)+v(z) \\
& \leq \frac{1}{2 \pi} \int_{-\pi}^{\pi} u\left(r e^{i \omega}+\rho e^{i \psi}\right) d \psi+\frac{1}{2 \pi} \int_{-\pi}^{\pi} v\left(r e^{i \omega}+\rho e^{i \psi}\right) d \psi
\end{aligned}
$$

is true. Denoting by $\nu$ the Riesz measure of the subharmonic function $v(z)$ we find its local potential $V_{\rho}\left(r e^{i \omega}\right)$ defined by (3). The latter inequality and the Poisson-Jensen formula imply

$$
u\left(r e^{i \omega}\right) \leq \frac{1}{2 \pi} \int_{-\pi}^{\pi} u\left(r e^{i \omega}+\rho e^{i \psi}\right) d \psi+V_{\rho}\left(r e^{i \omega}\right) .
$$

Fixing a point $r e^{i \theta}$ in the domain of definition of the function $u_{l}^{*}$ we consider a set $E \in \mathscr{E}(\theta, l)$ which provides the supremum in the definition of $u_{l}^{*}$, i.e.,

$$
u_{l}^{*}\left(r e^{i \theta}\right)=\frac{1}{2 \pi} \int_{E} u\left(r e^{i \omega}\right) d \omega .
$$

Integrating inequality (4) with respect to $\omega$ over $E$ we find

$$
u_{l}^{*}\left(r e^{i \theta}\right) \leq \int_{E}\left(\frac{1}{2 \pi} \int_{-\pi}^{\pi} u\left(r e^{i \omega}+\rho e^{i \psi}\right) d \psi\right) d \omega+\int_{E} V_{\rho}\left(r e^{i \omega}\right) d \omega .
$$


Since the measure of the set $E$ is equal to $2 \theta$ and the function $V_{\rho}\left(r e^{i \omega}\right)$ is even and does not increase for $\omega \in(0, \pi)$ for all sufficiently small $\rho$, we have the inequality

$$
\int_{E} V_{\rho}\left(r e^{i \omega}\right) d \omega \leq \int_{-\theta}^{\theta} V_{\rho}\left(r e^{i \omega}\right) d \omega .
$$

Hence,

$$
\begin{aligned}
u_{l}^{*}\left(r e^{i \theta}\right) \leq & \int_{E}\left(\frac{1}{2 \pi} \int_{-\pi}^{\pi} u\left(r e^{i \omega}+\rho e^{i \psi}\right) d \psi\right) d \omega \\
& +\int_{\theta}^{\theta} V_{\rho}\left(r e^{i \omega}\right) d \omega .
\end{aligned}
$$

To estimate the integral

$$
\int_{E}\left(\frac{1}{2 \pi} \int_{-\pi}^{\pi} u\left(r e^{i \omega}+\rho e^{i \psi}\right) d \psi\right) d \omega
$$

we use the identity satisfied by every measurable function $g(z)$ :

$$
\begin{aligned}
\int_{\pi}^{\pi} g\left(r e^{i \omega}+\rho e^{i \psi}\right) d \psi= & \int_{0}^{\pi} g\left(r e^{i \omega}+\rho e^{i(\omega+\psi)}\right) d \psi \\
& +\int_{0}^{\pi} g\left(r e^{i \omega}+\rho e^{i(\omega-\psi)}\right) d \psi \\
= & \int_{0}^{\pi}\left\{g\left(r(\psi) e^{i \omega+\alpha(\psi)}\right)+g\left(r(\psi) e^{i \omega-\alpha(\psi)}\right)\right\} d \psi .
\end{aligned}
$$

Here $r(\psi)=\left|r+\rho e^{i \psi}\right|, \alpha(\psi)=\arg \left(r+\rho e^{i \psi}\right)$. (Note that $\alpha(\psi)$ tends to zero uniformly with respect to $\psi$ as $\rho \rightarrow 0$.) Applying this identity to the function $u(z)$ we get

$$
\begin{aligned}
& \int_{E}(\left.\frac{1}{2 \pi} \int_{-\pi}^{\pi} u\left(r e^{i \omega}+\rho e^{i \psi}\right) d \psi\right) d \omega \\
& \quad=\frac{1}{2 \pi} \int_{0}^{\pi}\left(\int_{E-\alpha(\psi)} u\left(r(\psi) e^{i \omega}\right) d \omega+\int_{E+\alpha(\psi)} u\left(r(\psi) e^{i \omega}\right) d \omega\right) d \psi \\
& \quad=\frac{1}{2 \pi} \int_{0}^{\pi}\left(\int_{A} u\left(r(\psi) e^{i \omega}\right) d \omega+\int_{B} u\left(r(\psi) e^{i \omega}\right) d \omega\right) d \psi .
\end{aligned}
$$

Here $A \in \mathscr{E}(\theta-\alpha(\psi), l), B \in \mathscr{E}(\theta+\alpha(\psi), l)$ are the sets whose existence follows from Lemma 1 for all sufficiently small $\rho$. It follows from the definition of $u_{l}^{*}\left(r e^{i \gamma}\right)$ that

$$
\begin{aligned}
& \int_{A} u\left(r(\psi) e^{i \omega}\right) d \omega \leq u_{l}^{*}\left(r(\psi) e^{i(\theta-\alpha(\psi))}\right), \\
& \int_{B} u\left(r(\psi) e^{i \omega}\right) d \omega \leq u_{l}^{*}\left(r(\psi) e^{i(\theta+\alpha(\psi))}\right),
\end{aligned}
$$

for $\gamma=\theta \pm \alpha(\psi)$. Applying identity (8) to the function $u_{l}^{*}\left(r e^{i \theta}\right)$ and using relations (9), (10), and (11) we get

$$
\int_{E}\left(\frac{1}{2 \pi} \int_{-\pi}^{\pi} u\left(r e^{i \omega}+\rho e^{i \psi}\right) d \psi\right) d \omega \leq \frac{1}{2 \pi} \int_{-\pi}^{\pi} u_{l}^{*}\left(r e^{i \omega}+\rho e^{i \psi}\right) d \psi
$$


It follows from the Poisson-Jensen formula that

$$
\frac{1}{2 \pi} \int_{-\pi}^{\pi} v\left(z+\rho e^{i \psi}\right) d \psi-v(z)=V_{\rho}(z) .
$$

Setting $z=r e^{i \omega}$ in the latter equation and integrating it with respect to $\omega$ over the interval $(-\theta, \theta)$ we get

$$
\frac{1}{2 \pi} \int_{-\pi}^{\pi} \tilde{v}\left(r e^{i \theta}+\rho e^{i \psi}\right) d \psi-\tilde{v}\left(r e^{i \theta}\right)=\int_{\theta}^{\theta} V_{\rho}\left(r e^{i \omega}\right) d \omega .
$$

Using this identity we obtain from (7) and (12) the inequality

$$
u_{l}^{*}\left(r e^{i \theta}\right)+\tilde{v}\left(r e^{i \theta}\right) \leq \frac{1}{2 \pi} \int_{-\pi}^{\pi}\left\{u_{l}^{*}\left(r e^{i \theta}+\rho e^{i \psi}\right)+\tilde{v}\left(r e^{i \theta}+\rho e^{i \psi}\right)\right\} d \psi .
$$

In other words, the function $u_{i}^{*}(z)+\tilde{v}(z)$ satisfies the required integral inequality. This inequality together with continuity of the function $u_{l}^{*}(z)+\tilde{v}(z)$ completes the proof of Theorem 1.

\section{Conjecture of Weitsman}

Let $\Omega$ be a domain in the complex plane $C$ and let $G_{\Omega}(z, \zeta)$ be its Green function (defined by zero outside $\Omega \times \Omega$ as above). We assume that $\Omega$ satisfies the following conditions:

(1) $1 \in \Omega$;

(2) every closed arc of the angular measure $2 l$ of every circle with the center zero contains at least one point from $\mathrm{C} \backslash \Omega$.

Using Theorem 1 we will prove the following statement.

Theorem 2 (Weitsman's Conjecture). Lèt $\Omega$ be a domain in $\mathbf{C}$ satisfying conditions (1) and (2) and let $G_{\Omega}(z, \xi)$ be its Green function. The following inequalities hold for every $r>0$ :

$$
\begin{gathered}
\max _{|z|=r} G_{\Omega}(z, 1) \leq \max _{|z|=r} G_{\Omega_{0}}(z, 1), \\
\int_{-\pi}^{\pi} G_{\Omega}\left(r e^{i \varphi}, 1\right) d \varphi \leq \int_{-\pi}^{\pi} G_{\Omega_{0}}\left(r e^{i \varphi}, 1\right) d \varphi,
\end{gathered}
$$

where $\Omega_{0}=\{z: \arg (z)<l\}$ is the sector of angular opening $2 l$. (Here we assume that the Green function is equal to zero outside $\Omega \times \Omega$.)

Proof. First we prove inequality (13). Let

$$
u(z)=G_{\Omega^{\prime}}(z, 1), \quad z \in \mathbb{C},
$$

where $\Omega^{\prime}$ is an arbitrary domain compactly contained in $\Omega$ that has a continuous Green function. The function $u(z)$ admits the representation

$$
u(z)=u_{+}(z)-\log |1-z|,
$$

where $u_{+}(z)$ is a subharmonic function in $\mathbb{C}$. According to Example 2 the function $\log |1-z|$ has the local bell-type potential. Hence, we can apply Theorem 1 to the function $u(z)$. According to this theorem the function

$$
u_{l}^{*}\left(r e^{i \theta}\right)+\int_{-\theta}^{\theta} \log \left|1-r e^{i \omega}\right| d \omega, \quad z=r e^{i \theta},
$$


is subharmonic in the domain $\{z: \arg (z) \in(0, l)\}$. We denote by $g(z)$ the function

$$
g(z)=G_{\Omega_{0}}(z, 1)=g_{+}(z)-\log |1-z| .
$$

Obviously,

$$
\begin{aligned}
g_{l}^{*}\left(r e^{i \theta}\right) & =\tilde{g}\left(r e^{i \theta}\right)=\int_{-\theta}^{\theta} g\left(r e^{i \varphi}\right) d \varphi \\
& =\int_{-\theta}^{\theta} g_{+}\left(r e^{i \varphi}\right) d \varphi-\int_{-\theta}^{\theta} \log \left|1-r e^{i \varphi}\right| d \varphi .
\end{aligned}
$$

Since $g_{+}(z)$ is a harmonic function in $\Omega_{0}$ then it is easy to see that the function

$$
g_{l}^{*}\left(r e^{i \theta}\right)+\int_{-\theta}^{\theta} \log \left|1-r e^{i \varphi}\right| d \varphi
$$

is harmonic in the angle $\{z: \arg (z) \in(0, l)\}$. Summing up these arguments we conclude that the function

$$
f(z)=u_{l}^{*}(z)-g_{l}^{*}(z)
$$

is subharmonic in the angle $\{z: \arg (z) \in(0, l)\}$. Now we continue the function $f(z)$ in the double angle $\{z: \arg (z) \in(0,2 l)\}$ by the equation

$$
f\left(z^{*}\right)=f(z)
$$

(here $z^{*}$ is a point symmetric to $z$ with respect to the ray $\{z: \arg (z)=l\}$ ). We need to show that the continued function $f(z)$ is subharmonic in the double angle $\{z: \arg (z) \in(0,2 l)\}$. Indeed, it follows from the definition of (15) and from the properties of the domain $\Omega$ that the function $u(z)$ satisfies the conditions of Remark 1. Hence, the function $u_{i}^{*}\left(r e^{i \theta}\right)$ does not depend on $\theta$ in some neighborhood of the ray $\{\theta=l, r>0\}$. According to the definition of the function $g_{l}^{*}(z)$ we have $\partial g\left(r e^{i \theta}\right) / \partial \theta=0$ for $\theta=l$ and $r>0$. So we get

$$
\left.\frac{\partial\left(u_{l}^{*}\left(r e^{i \theta}\right)-g_{l}^{*}\left(r e^{i \theta}\right)\right)}{\partial \theta}\right|_{\theta=l}=0 .
$$

It follows from this relation that the continued function $f(z)$ is subharmonic in $\{z: \arg (z) \in(0,2 l)\}$.

Since the function $f(z)$ is bounded in $\{z: \arg (z) \in(0,2 l)\}$ and vanishes on the boundary of this angle then, according to the Phragmén-Lindelöf theorem, this function satisfies the inequality $f(z) \leq 0$ inside this angle.

To complete the proof of inequality (13) we use the following double inequality $(\alpha \in(0,2 \pi])$ :

$$
0 \leq \frac{1}{2 \eta} f\left(r e^{i \eta}\right) \leq \frac{1}{2 \eta} \int_{-\eta}^{\eta} u\left(r e^{i \alpha+i \varphi}\right) d \varphi-\frac{1}{2 \eta} \int_{-\eta}^{\eta} g\left(r e^{i \varphi}\right) d \varphi .
$$

Passing to the limit as $\eta \rightarrow 0$ we obtain

$$
u\left(r e^{i \alpha}\right) \leq g(r) \quad \forall \alpha \in(0,2 \pi] .
$$

It is easy to see that $u\left(r e^{i \alpha}\right)=G_{\Omega^{\prime}}\left(r e^{i \alpha}, 1\right)$ and $g(r)=\max G_{\Omega_{0}}\left(r e^{i \alpha}, 1\right)$ for $\alpha \in(0,2 \pi]$. Hence we have the inequality

$$
\max _{|z|=r} G_{\Omega^{\prime}}(z) \leq \max _{|z|=r} G_{\Omega_{0}}(z)
$$


Since the function $G_{\Omega}(z)$ can be approximated by the continuous functions $G_{\Omega^{\prime}}(z)$ it implies inequality (13).

Now we prove inequality (14). This proof will be almost the same as that of (13). We choose only suitable functions $u(z)$ and $g(z)$ and repeat the previous proof. Let $u(z)$ be defined by

$$
u(z)=\frac{1}{2 \pi} \int_{-\pi}^{\pi} G_{\Omega^{\prime}}\left(z, R e^{i \lambda}\right) d \lambda,
$$

where $\boldsymbol{\Omega}^{\prime}$ is a domain compactly contained in $\boldsymbol{\Omega}$ and having a continuous Green function. Obviously, the conditions of Remark 1 are satisfied for this function and, moreover, the following representations holds:

$$
u(z)=u_{+}(z)-v(z) \text {, }
$$

where $u_{+}(z)$ is a subharmonic function on $\mathbf{C}$ and $v(z)$ is represented by the formula

$$
v(z)=\frac{1}{2 \pi} \int_{-\pi}^{\pi} \log \left|R e^{i \lambda}-z\right| d \lambda .
$$

Let $g(z)$ be defined by the equation

$$
g(z)=\frac{1}{2 \pi} \int_{-\pi}^{\pi} G_{\Omega_{0}}\left(z, R e^{i \lambda}\right) d \lambda=\frac{1}{2 \pi} \int_{-l}^{l} G_{\Omega_{0}}\left(z, R e^{i \lambda}\right) d \lambda .
$$

We use the representation of this function

$$
g(z)=g_{+}(z)-v(z),
$$

where $v(z)$ is defined by (16). It is easy to see that the function $g_{+}(z)$ is harmonic in the domain $\Omega_{0}$. Note also that the function $g\left(r e^{i \phi}\right)$ is even with respect to $\varphi$ for every $r>0$ and does not increase on $(0, \pi)$. Hence, the following representation for $g_{l}^{*}\left(r e^{i \theta}\right)$ is valid:

$$
g_{l}^{*}\left(r e^{i \theta}\right)=\tilde{g}\left(r e^{i \theta}\right)=\int_{-\theta}^{\theta} g\left(r e^{i \varphi}\right) d \varphi .
$$

Now repeating the proof of (13) we obtain

$$
\max _{\alpha \in(0,2 \pi]} u\left(e^{i \alpha}\right) \leq g(1),
$$

so,

$$
\int_{-\pi}^{\pi} G_{\Omega^{\prime}}\left(1, R e^{i \varphi}\right) d \varphi \leq \int_{-\pi}^{\pi} G_{\Omega_{0}}\left(1, R e^{i \varphi}\right) d \varphi .
$$

In order to complete the proof, we use the beautiful property of Green functions that $G(z, \xi)=G(\xi, z)$ and approximate the Green function $G_{\Omega}(z, \zeta)$ by the continuous one. In such a way we deduce inequality (14) from (17). Theorem 2 is proved.

Now Lemma 1 will be proved.

\section{Proof of Lemma 1}

Let $E \in \mathscr{E}(\theta, l), \theta<l \leq \pi$. According to the definition of the family $\mathscr{E}(\theta, l)$ we have $\operatorname{diam}(E) \leq 2 l$ and $\operatorname{mes}(E)=2 \theta$. It is convenient to consider 
the image of $E$ under the mapping $F: \mathbf{R} \rightarrow \mathbf{R} / 2 \pi \mathrm{N}$ of the real axis onto the unit circle $T$ instead of the set $E \subset \mathbf{R}$. We denote this image by $E$ as well. All metric characteristics of the set $E$ on the circle are the corresponding characteristics of the set $F^{-1}(E)$ on the real axis. For example, diam $(E)$ is the lower bound of mes $(I)$ over all arcs $I \supset E$, where $\operatorname{mes}(I)$ is the Lebesgue measure on $T$ normalized by the relation $\operatorname{mes}(T)=2 \pi$. We suppose that $A \simeq B$ if $\operatorname{mes}(A \backslash B)=\operatorname{mes}(B \backslash A)=0$.

We say that $E \in \mathscr{E}(\theta, l)$ is

a set of type 1 if $E \simeq I$ for some interval (arc);

a set of type 2 if $E \simeq I_{1} \cup I_{2}$ for some intervals $I_{1}, I_{2}$ and $E$ is not a set of type 1;

a set of type 3 if $E$ is not a set of type 1 or 2 .

First let $E$ be a set of type 1 . In this case we set

$$
A=E_{\varepsilon} \cup E_{-\ell}, \quad B=E_{\ell} \cap E_{-\ell},
$$

where $E_{\varepsilon}$ is the $\varepsilon$-shift of the set $E$, i.e., $E_{\varepsilon}=\{\varphi+\varepsilon: \varphi \in E\}$. Since the measure of the set $E$ equals $2 \theta<2 l$ and coincides with its diameter, then $\operatorname{diam}(A)$ and $\operatorname{diam}(B)$ are not larger than $2 l$ for all sufficiently small $\varepsilon>0$. Thus the assertion of Lemma 1 for sets of type 1 is proved.

Now let $E$ be a set of type 2 or 3 . At first we prove that the inequality

$$
\left|E_{\varepsilon} \cap E_{-\varepsilon}\right| \leq|E|-4 \varepsilon
$$

is satisfied for all sufficiently small $\varepsilon>0$. It is easy to see that inequality (18) is valid for all sets of type 2. Indeed, in this case both parts of inequality (18) coincide for all sufficiently small $\varepsilon>0$. If $E$ is a set of type 3 then there exist two reciprocally alternate sets $\left\{a_{1}, a_{2}, a_{3}\right\}$ and $\left\{b_{1}, b_{2}, b_{3}\right\}$ on the circle such that all $b_{i}$ are points of density of $E$ and $a_{i}$ are points of density of the complement of $E$. Now we divide the circle into three $\operatorname{arcs} L_{i}=\left(c_{i}, d_{i}\right)$ in such a way to provide the condition: the interior of the arc $L_{i}$ contains the arc $\left[a_{i}, b_{i}\right]$ (without loss of generality we can assume that the arcs $\left[a_{i}, b_{i}\right]$ do not intersect). Denoting the indicator of the set $E$ by $\chi(t)$ we obtain the relations (19)

$$
\begin{aligned}
\int_{L_{i}} \chi(t+\varepsilon) \chi(t-\varepsilon) d t & \leq \int_{c_{i}}^{a_{i}} \chi(t+\varepsilon) d t+\int_{a_{i}}^{b_{i}} \chi(t-\varepsilon) d t+\int_{b_{i}}^{d_{i}} \chi(t+\varepsilon) d t \\
& =\int_{L_{i}} \chi(t+\varepsilon) d t-\int_{a_{i}}^{b_{i}} \chi(t+\varepsilon) d t+\int_{b_{i}}^{a_{i}} \chi(t-\varepsilon) d t \\
& =\int_{L_{i}} \chi(i+\varepsilon) d t+\int_{\left|t-a_{i}\right| \leq \varepsilon} \chi(t) d t-\int_{\left|t-b_{i}\right| \leq \varepsilon} \chi(t) d t .
\end{aligned}
$$

Since $a_{i}$ and $b_{i}$ are points of density of the sets $E^{0}$ and $E$ respectively then the inequalities

$$
\int_{\left|t-a_{i}\right| \leq e} \chi(t) d t-\int_{\left|t-b_{i}\right| \leq e} \chi(t) d t \leq-\frac{4 \varepsilon}{3}
$$

are valid for all sufficiently small $\varepsilon>0$. Summing inequalities (19) over $i$ and using (20) we get (18).

For the sake of definiteness we suppose that $E \subseteq[0,2 l]$. Due to this assumption there exists $\lambda \leq 2 l-\varepsilon$ such that the measure of the set

$$
C=\left\{E_{-\varepsilon} \backslash\left(E_{\varepsilon} \cap E_{-\varepsilon}\right)\right\} \cap[-\varepsilon, \lambda]
$$


is equal to $|E|-2 \varepsilon-\left|E_{\varepsilon} \cap E_{-\varepsilon}\right|$. According to inequality (18) the estimate $|C| \geq 2 \varepsilon$ is valid, and hence, $\lambda \geq \varepsilon$. Let

$$
B=\left(E_{\varepsilon} \cap E_{-\varepsilon}\right) \cup C, \quad A=\left(E_{\varepsilon} \cup E_{-\varepsilon}\right) \backslash C .
$$

It is easy to see that

$$
\chi_{E}(t+\varepsilon)+\chi_{E}(t-\varepsilon)=\chi_{A}(t)+\chi_{B}(t),
$$

$\operatorname{mes}(A)=2 \theta+2 \varepsilon, \operatorname{mes}(B)=2 \theta-2 \varepsilon ; \operatorname{diam}(A)$ and $\operatorname{diam}(B)$ do not exceed $2 l$. Since $A \subseteq[-\varepsilon, 2 l-\varepsilon], B \subseteq[\varepsilon, 2 l+\varepsilon]$, then Lemma 1 is proved.

\section{A genERAI VERSION OF THEOREM 1}

After reading the preprint of this paper Baernstein proposed that Theorem 1 implies the estimate.

$$
\int_{-\pi}^{\pi} \Phi\left(G_{\Omega}\left(r e^{i \varphi}, 1\right)\right) d \varphi \leq \int_{-\pi}^{\pi} \Phi\left(G_{\Omega_{0}}\left(r e^{i \varphi}, 1\right)\right) d \varphi
$$

as well (for every convex positive increasing function $\Phi$ ) and sketched the proof. The latter estimate implies, in particular, the corresponding inequality for $L_{p}$-norms on a circle:

$$
\int_{-\pi}^{\pi} G_{\Omega}^{p}\left(r e^{i \varphi}, 1\right) d \varphi \leq \int_{-\pi}^{\pi} G_{\Omega_{0}}^{p}\left(r e^{i \varphi}, 1\right) d \varphi, \quad p \in[1, \infty],
$$

and hence inequalities (13) and (14) established by Theorem 2 are particular cases of this inequality (for $L_{\infty}$ - and $L_{1}$-norms respectively). Unfortunately, his proof cannot be deduced from Theorem 1 directly. Here we propose a different proof of this inequality. We need a more general version of Theorem 1. Before formulating this theorem we introduce a notion of a bell-type symmetrization.

Let $E$ be an open set in the annulus $\Pi=\left\{z:|z| \in\left(r_{1}, r_{2}\right)\right\}$ and let $E^{*}$ be its circular symmetrization with respect to the ray $\{z: \operatorname{Im} z=0, \operatorname{Re} z>0\}$, i.e., for every $r \in\left(r_{1}, r_{2}\right)$ the intersection $\{|z|=r\} \cap E^{*}$ coincides with the arc $\left\{|z|=r: \arg z \in\left(-|E|_{r} / 2,|E|_{r} / 2\right)\right\},|E|_{r}$ being the Lebesgue measure of the intersection $\{|z|=r\} \cap E$ (we assume that this measure of the circle is equal to $2 \pi$ ). We will say $A$ is a "bell-symmetric" set if the set $A$ can be obtained by circular symmetrization of some suitable set $B$, i.e., $A=B^{*}$ for some $B$.

Let $\nu$ be a measure on the annulus $\Pi$. Setting

$$
\stackrel{\circ}{\nu}(A)=\sup \left\{\nu(E): E^{*}=A\right\}, \quad \stackrel{\circ}{\nu}(E)=\stackrel{\circ}{\nu}(\bar{E}), \quad \bar{E}=\{\bar{z} \in E\},
$$

for every bell-symmetric set $A$ and continuing this additive function onto the minimal $\sigma$-algebra generated by all bell-type symmetric sets we define a new measure $\stackrel{\nu}{\nu}$. This measure is called a circular symmetrization of the measure $\nu$.

We will say that a continuous function $f(z)$ defined on the annulus $\Pi$ is "bell-type" if $f(z) \equiv f(\bar{z})$ and $f\left(r e^{i \alpha}\right) \leq f\left(r e^{i \beta}\right)$ for every $\alpha \geq \beta, \alpha, \beta \in$ $[0, \pi]$. Let $g(z)$ be a continuous function. We say that a bell-type function $\stackrel{\circ}{g}(z)$ is a circular symmetrization of the continuous function $g(z)$ if $\stackrel{\circ}{g}^{*} \equiv g^{*}$, where * denotes Baernstein's operator. (It is useful to recall that

$$
f^{*}\left(r e^{i \theta}\right)=\sup _{|E|=2 \theta} \int_{E} f\left(r e^{i \varphi}\right) d \varphi
$$


for $f$ measurable in the sense of Borel, $|\cdot|$ denotes the Lebesgue measure on $\mathbb{R}$; see [1].)

Considering the measures $\nu$ and $i$ as continuous linear functionals in the space of continuous functions with compact supports and using these new notions, we can define a functional $i(f)$ for $f$ an arbitrary nonnegative bell-type function by

$$
\stackrel{\circ}{\nu}(f)=\sup \left\{\nu(g): g^{*}=f^{*}\right\} .
$$

It is obvious that there exists only one linear functional $\stackrel{\nu}{\nu}$ satisfying the latter relation and the additional conditions

$$
\stackrel{\circ}{\nu}(f(z))=\stackrel{\circ}{\nu}(f(\bar{z})) \text {. }
$$

It is easy to show the latter defintion of the measure $\stackrel{i}{\nu}$ is equivalent to the previous one. Now we give some examples.

Example 3. Let $W$ be a measurable set on the circle $|z|=r$ and let $\nu$ be the uniform probability distribution supported by $W$. Then $\nu$ is the uniform probability distribution supported by the $\operatorname{arc}\{|z|=r, \arg z \in(-|W| / 2,|W| / 2)\}$.

Example 4. If $\nu$ is a discrete measure, then $\stackrel{\circ}{\nu}$ is discrete as well. It is easy to see that the support of the measure $i$ is contained in the nonnegative real axis. Indeed, let $z_{k}$ be a complex sequence supported by the measure $\nu$ and $\nu\left(\left\{z_{k}\right\}\right)=p_{k}$. To define $\stackrel{\circ}{\nu}$ it is sufficient to set

$$
\stackrel{\circ}{\nu}(A)=\sum_{\left|z_{k}\right| \in A} p_{k}
$$

for every measurable set $A$.

The following property of the operator $\left(^{\circ}\right)$ will be extremely useful for its applications.

Let $\nu$ be a nonnegative measure and let $\nu_{t}$ be its convolution with the uniform distribution supported by the disk $|z|<t$. It is easy to see that $\nu_{t}$ tends weakly to $\nu$ as $t \rightarrow 0$. Moreover, the following lemma is true.

Lemma 2. Let $\nu$ be a nonnegative measure on $\mathbb{C}$ and $\nu_{t}$ the measure defined above. The measure $\left(\nu_{t}\right)^{\circ}$ converges weakly to ${ }^{\circ}$ as $t \rightarrow 0$.

Remark 2 . The family $\nu_{t}$ cannot be replaced by an arbitrary family $\nu_{t}$ which converges weakly to $\nu$ ! Indeed, if $\nu_{t}$ is a family of discrete measures converging weakly to a uniform distribution on a symmetric arc, then the family of $\left(\nu_{t}\right)^{\circ}$ converges weakly to some single-point measure.

Proof of Lemma 2. It is sufficient to verify weak convergence only for all nonnegative bell-type functions $f$ having a compact support. We have the estimate $\stackrel{\circ}{\nu}(f)-\stackrel{\circ}{\nu}_{t}(f) \leq \sup \left\{v(g): g^{*}=f^{*}\right\}-\sup \left\{\nu_{t}(g): g^{*}-f^{*}\right\} \leq \varepsilon+\nu\left(g_{\varepsilon}\right)-\nu_{t}\left(g_{\varepsilon}\right)$, for every $\varepsilon>0, g_{\varepsilon}$ being a suitable approximating function for $\stackrel{\nu}{\nu}(f)$. It follows from this inequality that

$$
\lim \sup \dot{\nu}_{t}(f) \geq \stackrel{i}{\nu}(f) \text {. }
$$


Now we will get the inverse estimate. We have

$$
\stackrel{\circ}{\nu}_{t}(f)-\stackrel{\circ}{\nu}(f)=\sup \left\{\nu_{t}(g): g^{*}=f^{*}\right\}-\sup \left\{\nu(g): g^{*}=f^{*}\right\} .
$$

Unfortunately, we cannot assert that the first member of the right-hand side coincides with the value of $\nu_{t}$ at some suitable continuous functions, but such a measurable function $g_{t}$ exists. Moreover, the function $g$ belongs to a space $L^{\infty}(K)$, where $K$ is some disk containing $\operatorname{supp}(f)$, and $g^{*}=f^{*}$. Indeed, the family of continuous functions $g$ satisfying $g^{*}=f^{*}$ is contained in the ball $\|x\| \leq\|f\|$ of the space $L^{\infty}(K)$ and, hence, is a weakly precompact set (we consider $L^{\infty}(K)$ as the space of all continuous linear functionals on $L^{1}(K)$ ). Due to the absolute continuity of the measure $\nu_{t}$ with respect to the Lebesgue measure, the density of $\nu_{t}$ belongs to $L^{1}(K)$. Thus, $\nu_{t}(g)$ is the value of the continuous linear functional $g \in L^{\infty}(K)$ at $d \nu_{t} / d m \in L^{1}(K), m$ being the Lebesgue measure on $\mathrm{C}$. Hence the function $g_{t} \in L^{\infty}$ exists.

Note that in our arguments we use the absolute continuity of the measure $\nu_{t}$ with respect to the Lebesgue measure.

It remains to prove that $g_{t}^{*}=f^{*}$. The latter equation is fulfilled for all prelimit functions $g$. Using the same arguments as above we can prove that all prelimit functions form a weak precompact set of the space $L^{\infty}(T)$, where $T$ is an arbitrary circle. Thus, the convergence $\int_{E} g \rightarrow \int_{E} g_{t}$ holds for every measurable set $E$ on this circle. If $E$ is a set such that $\int_{E} g_{t}=g_{i}^{*}\left(r e^{i \theta}\right)=g_{i}^{*}$, $|E|=2 \theta$, then we have

$$
f^{*}=g^{*} \leq g_{t}^{*} \text {. }
$$

With $E$ satisfying $\int_{E} f=f^{*}\left(r e^{i \theta}\right)=f^{*},|E|=2 \theta$, we have

Thus $f^{*}=g_{i}^{*}$.

$$
f^{*}=g^{*} \geq g_{t}^{*} \text {. }
$$

It remains to estimate $i_{t}(f)-\stackrel{\nu}{\nu}(f)$ from above. We have

$$
\stackrel{\circ}{\nu}_{t}(f)-\stackrel{\circ}{\nu}(f) \leq \nu_{t}\left(g_{t}\right)-\nu(u),
$$

where $g_{t}$ had been defined above, $u$ is an arbitrary function satisfying the equation $f^{*}=u^{*}$. Obviously, we can suppose that $g_{t}$ belongs to $L^{\infty}(K, \nu)$ as well. It remains to choose a suitable function $u$ and to make a good estimate. Rewrite the right side of the latter inequality as

$$
\int g_{t} * \beta_{t} d \nu-\int u d \nu,
$$

where $g_{t} * \beta_{t}$ is the convolution of the function $g$ with uniform probability distribution to the disk $|z| \leq t$. Note, that $g_{t}$ belongs to $L^{\infty}(K, \nu+m), m$ the Lebesgue measure on $\mathbf{C}$, and, moreover, to the ball of radius $\|f\|$ of this space due to the inequality

$$
\sup \left\{g_{\imath}(z): z \in \mathbb{C}\right\}=\sup \{f(z): z \in \mathbb{C}\}
$$

Hence $g_{t}$ has a weak-limit point $g_{0} \in L^{\infty}(K, \nu+m)$. Since the set of functions $g_{t}$ satisfying $g_{t}^{*}=f^{*}$ is weakly closed (see the proof of the equation $g_{t}^{*}=f^{*}$ ), we have $f^{*}=g_{t}^{*}=g_{0}^{*}$. Obviously, $g_{t} * \beta_{t}$ has the weak-limit point $g_{0}$ as well due to the weak convergence $g_{0} * \beta_{t} \rightarrow g_{0}$ as $t \rightarrow 0$. Thus, we have the estimate

$$
\stackrel{\circ}{\nu}_{t}(f)-\stackrel{\circ}{\nu}(f) \leq \int g_{t} * \beta_{t} d \nu-\int g_{0} d \nu=o(t) \text { as } t \rightarrow 0 .
$$

Lemma 2 is proved. 
Let $v(z)$ be a subharmonic function in the annulus $\Pi$ and let $\nu$ be its Riesz measure. We need the following definition.

Definition. A function $\stackrel{\circ}{v}(z)$ being subharmonic in the annulus $\Pi$ an having a Riesz measure $\mu$ is called a bell-type symmetrization of a subharmonic function $v(z)$ if their Riesz measures are connected by the relation $\mu=\nu$.

Now we state the general version of Theorem 1.

Theorem 3. Let $u(z)$ be a subharmonic function represented by the difference (1) in the annulus $\left\{|z| \in\left(r_{1}, r_{2}\right)\right\}$. If $\stackrel{\circ}{v}(z)$ is a bell-type symmetrization of $v(z)$, then the function $u_{l}^{*}\left(r e^{i \theta}\right)+\int_{-\theta}^{+\theta} \stackrel{\circ}{v}\left(r e^{i \varphi}\right) d \varphi$ is continuous and subharmonic in the annular sector $\left\{z=r e^{i \varphi}: r \in\left(r_{1}, r_{2}\right), \theta \in(0, l)\right\}$.

Proof. In order to prove Theorem 3 it is sufficient in the proof of Theorem 1 to replace inequality (6) by

$$
\int_{E} V_{\rho}\left(r e^{i \omega}\right) d \omega \leq \int_{-\theta}^{\theta} \stackrel{\circ}{V}_{\rho}\left(r e^{i \omega}\right) d \omega,
$$

where $\stackrel{\circ}{V}_{\rho}$ is the local potential of the subharmonic function $\stackrel{\circ}{v}(z)$. Thus, it remains to prove the latter inequality.

To prove inequality (22) we consider the subharmonic function

$$
v_{E}(z)=\int_{E} v\left(z e^{i \omega}\right) d \omega .
$$

This function has a Riesz measure $\mu$ represented as

$$
\mu(B)=\int_{E} \nu\left(B e^{i \omega}\right) d \omega,
$$

$\nu$ being the Riesz measure of $v$. Let $\stackrel{\circ}{v}$ be a bell-type symmetrization of $v$ with Riesz measure $i$. It is easy to see that the local potential of $v_{E}(z)$ at $z=r$ is equal to the left-hand side of (22). On the other hand, the subharmonic function

$$
{\stackrel{\circ}{v^{*}}}(z)=\int_{E^{*}} \stackrel{i}{ }\left(z e^{i \omega}\right) d \omega
$$

has at the point $z=r$ a local potential coinciding with the right-hand side of (22). Since a local potential of positive Riesz measure $\lambda$ at $z=r$ can be represented as

$$
\iint_{|\xi|<\rho} \log \frac{\rho}{|\xi|} d \lambda(\xi+r)=\int_{0}^{\rho} R(t)\left(\int_{B_{t}} d \lambda(\xi+r)\right) d t,
$$

for a suitable positive function $R(t), B_{t}$ being the disk $|z|<\rho$, then to prove (22) it is sufficient to verify the inequality

$$
\int_{B_{t}} d \nu_{E}(\xi+r) \leq \int_{B_{t}} d \nu_{E} \cdot(\xi+r)
$$

or

$$
\int_{E} \nu\left(B_{t}+r e^{i \psi}\right) d \psi \leq \int_{-|E| / 2}^{|E| / 2} \stackrel{\circ}{\nu}\left(B_{t}+r e^{i \psi}\right) d \psi
$$


In proving the latter inequality we can suppose that the measure $\nu$ is continuous with respect to the Lebesgue measure in $\mathbb{C}$ and has a density $\mu(z)$. This assumption does not restrict generality as follows from Lemma 2. It is easy to see that the measure $i$ has the density $\lambda$ such that $\lambda^{*}=\mu^{*}$. Thus the latter inequality can be obtained from such an inequality, $|E|=\theta$,

$$
\int_{E}\left(\int_{-\pi}^{\pi}\left(\mu\left(\rho e^{i \omega}+r e^{i \psi}\right) d \omega\right) d \psi \leq \int_{-\pi}^{\pi} \mu^{*}\left(\rho e^{i \omega}\right) d \omega .\right.
$$

The proof of this inequality is the same as inequality (12). Theorem 3 is now proved.

In order to get the general inequality (21) we prove first that

$$
G_{\Omega}^{*}\left(1, r e^{i \theta}\right) \leq G_{\Omega_{0}}^{*}\left(1, r e^{i \theta}\right)
$$

for every $r \in\left(r_{1}, r_{2}\right), 0 \leq \theta \leq 2 \pi$, where * is the usual transform of Baernstein [1], i.e.,

$$
f^{*}\left(r e^{i \theta}\right)=\sup \left\{\int_{E} f\left(r e^{i \varphi}\right) d \varphi:|E|=2 \theta\right\}, \quad 0 \leq \theta \leq 2 \pi, r \in\left(r_{1}, r_{2}\right),
$$

for every measurable function $f$. The latter inequality immediately implies (22) (see, for example, [1]).

It is easy to see that the right-hand side of (23) is equal to

$$
\int_{-\theta}^{\theta} G_{\Omega_{0}}\left(1, r e^{i \varphi}\right) d \varphi
$$

and hence it is sufficient to prove that

$$
\int_{E} G_{\Omega}\left(1, r e^{i \varphi}\right) d \varphi \leq \int_{-\theta}^{\theta} G_{\Omega_{0}}\left(1, r e^{i \varphi}\right) d \varphi
$$

for every measurable set $E$ such that $|E|=2 \theta$. In order to get the latter inequality we repeat the proof of Theorem 2 (using Theorem 3 instead of Theorem 1) for a pair of $\delta$-subharmonic functions

$$
u(z)=\int_{E} G_{\Omega}\left(z, R e^{i \lambda}\right) d \lambda \text { and } g(z)=\int_{-|E| / 2}^{|E| / 2} G_{\Omega_{0}}\left(z, R e^{i \lambda}\right) d \lambda,
$$

where $\theta=|E| / 2$. Noting that in the representations for $u(z)$ and $g(z)$ by the differences

$$
\begin{gathered}
u(z)=u_{+}(z)-\int_{E} \log \left|R e^{i \lambda}-z\right| d \lambda=u_{+}(z)-u_{-}(z), \\
g(z)=g_{+}(z)-\int_{-|E| / 2}^{|E| / 2} \log \left|R e^{i \lambda}-z\right| d \lambda=g_{+}(z)-g_{-}(z),
\end{gathered}
$$

the function $g_{-}(z)$ is the bell-type symmetrization of the function $u_{-}(z)$, it is easy to obtain the inequality

$$
\int_{E} G_{\Omega}\left(z, r e^{i \varphi}\right) d \varphi \leq \int_{-\theta}^{\theta} G_{\Omega_{0}}\left(1, r e^{i \varphi}\right) d \varphi,
$$

for every set $E,|E|=2 \theta$, (similar to inequality (14) in the proof of Theorem 2). Inequality (23) and hence (21) are proved.

Now we define a notion of $l$-bounded bell-type symmetrization and say a few words about possible generalizations and modifications of Theorem 3. 
Definition. Let $E$ be a measurable set in the annulus $\Pi$. A set $E_{l}^{*}$ is called an $l$-bounded circular symmetrization of the set $E$ (with respect to the positive ray) if the intersection $E_{l}^{*}$ with every circle $|z|=r, r \in\left(r_{1}, r_{2}\right)$, is an arc $(-\alpha, \alpha)$, where

$$
2 \alpha=\sup \left\{m_{r}(E \cap I): I \text { being an arc such that } m_{r}(I)=2 \alpha\right\}
$$

(here $m_{r}$ is Lebesgue measure on the circle $|z|=r$ such that $m_{r}\{|z|=r\}=$ $2 \pi)$.

Using this notion we can define such a notion as an l-bounded bell-type symmetric set and an l-bounded bell-type symmetrization of measures or subharmonic functions similar to corresponding notions linked with the usual circular symmetrization. Obviously, the following relations hold: $E_{l}^{*} \subseteq E^{*}$, $E_{l}^{*} \subseteq\{z \in \Pi: \arg z \in(-l, l)\}, \stackrel{\circ}{\mu}_{l} \leq \stackrel{\circ}{\mu}$, at least for all bell-type symmetric sets, $\stackrel{\circ}{\mu}$ and $\stackrel{\circ}{\mu}_{l}$ being the bell-type symmetrization and the l-bounded belltype symmetrization of a nonnegative measure $\mu$ respectively. However, the following proposition is true.

Theorem 4. If the conditions of Theorem 3 are satisfied then the function $u_{i}^{*}\left(r e^{i \theta}\right)$ $+\int_{-\theta}^{+\theta} \dot{o}_{l}\left(r e^{i \varphi}\right) d \varphi$ is continuous and subharmonic in the annulus sector $\{z=$ $\left.r e^{i \varphi}: r \in\left(r_{1}, r_{2}\right), \theta \in(0, l)\right\}$, where $\dot{v}_{l}$ is the l-bounded bell-type symmetrization of the subharmonic function $v$ from the representation (1).

The proof of this Theorem is the same as the proof of Theorem 3.

Other possible modifications of Theorem 3 are linked with applications of Theorem 4 for periodic $\delta$-subharmonic functions defined on a strip $|\operatorname{Im} z| \epsilon$ $(a, b)$ and various limits as periods tend to infinity. We do not formulate these results here.

I would like to thank Professor A. Eremenko for information on this problem. I also thank Professor A. Baernstein for discussions on the first version of this paper. Due to these discussions Theorems 3 and 4 appeared. I thank Professor A. Weitsman for corrections in this paper.

\section{REFERENCES}

1. A. Baernstein II, Integral means, univalent functions and circular symmetrization, Acta Math. 133 (1974), 139-169.

2. __, An extremal problem for certain subharmonic functions in the plane, Rev. Mat. Iberoamericana 4 (1988), 199-218.

3. A. E. Fryntov, One extremal problem of potential theory, Soviet Math. Dokl. 37 (1988), 754-755.

4. W. K. Hayman and P. B. Kennedy, Subharmonic functions, Academic Press, San Diego, 1976.

5. A. Weitsman, Some remarks on the spread of a Nevanlinna deficiency, Mittag-Leffler Technical report No. 7, 1977.

Department of MAThematics, Institute for Low Temperature and ENgINeering, KHARKOV 310164, UKRAINE

E-mail address: fryntovemath25.ilt.kharkov.ua 